\title{
O EFEITO DA REDUÇÃO DE ÁGUA NA LAVAGEM DA POLPA KRAFT NO BRANQUEAMENTO ECF
}

\author{
G. VENTORIM ${ }^{1}$, T. C. FRIGIERI ${ }^{2}$ e J. S. C. FAVARO ${ }^{2}$ \\ ${ }^{1}$ Universidade Estadual Paulista, Departamento de Engenharia Industrial Madeireira \\ 2 Universidade Estadual Paulista, Departamento de Engenharia Mecânica \\ E-mail para contato: ventorim@itapeva.unesp.br
}

\begin{abstract}
RESUMO - O objetivo do presente trabalho foi estudar o uso de efluente da lavagem do branqueamento ECF (Livre de cloro Molecular) da celulose, visando redução no consumo de água fresca, avaliando seu efeito na qualidade da polpa. Foi utilizada polpa de Eucalyptus spp. deslignificada com oxigênio. A mesma sequência de branqueamento, $\mathrm{D}(\mathrm{E}+\mathrm{P}) \mathrm{DP}$, foi realizada dez vezes, nas mesmas condições (temperatura, consistência e tempo). Para cada sequência, foi feita lavagem entre estágios com diferente fator de lavagem: 9, 6, 3 e $0 \mathrm{~m}^{3}$ de água destilada por tonelada de polpa, buscando atingir a alvura de $92 \pm 0,5 \%$ ISO. Os resultados mostraram o aumento da DQO por causa do acúmulo da matéria orgânica decorrente da reutilização dos efluentes das sequências anteriores. Este aumento da DQO fez com que os resultados da alvura baixassem durante os ciclos e também na redução da água de lavagem, tornando necessária a lavagem entre os estágios de branqueamento. Neste trabalho o resultado da lavagem até $3 \mathrm{~m}^{3} / \mathrm{t}$ de polpa foi tolerável e recomendável.
\end{abstract}

\section{INTRODUÇÃO}

O conceito sustentabilidade é uma preocupação mundial por causa da escassez dos recursos naturais que cresce a cada dia. Desta maneira, o ramo industrial está empenhado em desenvolver novas tecnologias de baixo impacto ambiental, que atendam às legislações ambientais, bem como às exigências de mercado e da qualidade do produto. O setor de branqueamento na indústria de celulose, dentre os diversos estágios que compõem o processo Kraft, a reutilização de águas e a conservação ou recuperação de substâncias é de grande interesse, pois é neste setor que se gera a maior carga de poluentes no efluente líquido (COSTA et al., 2006).

A reutilização do efluente do processo de branqueamento tem o nome de fechamento de circuito das águas, que significa a recirculação dos filtrados para a recuperação química, é a redução do fluxo utilizando-se sistemas eficientes de lavagem da polpa. Conforme Almeida et al. (2008) a lavagem entre os estágios de branqueamento, além de separar o material solubilizado da polpa e expor novas superfícies à ação dos reagentes de branqueamento, reduz desta forma, o consumo de reagentes utilizados no próximo estágio de branqueamento.

Orgânicos solúveis provenientes das matérias-primas e produtos químicos utilizados nos processos contribuem para uma maior Demanda Química de Oxigênio (DQO) e Compostos Halogenados Adsorvíveis (AOX) (Ranganathan et al., 2007). A redução do consumo de químicos, 
melhoramento da alvura da polpa, diminuição da carga de AOX no efluente e principalmente minimização do volume de efluentes a ser enviado para a estação de tratamento de efluentes (ETE) é o princípio da recirculação dos filtrados nas sequências de branqueamento (Thakore et al., 2001).

O payback, como o período de recuperação de um investimento da prensa na planta de branqueamento, consiste na identificação do prazo em que o montante do dispêndio de capital efetuado seja recuperado por meio de fluxos líquidos de caixa gerados pelo investimento (Kassai et $a l ., 2000)$. A redução do consumo de água visa resolver não somente interesses ambientais, mas também para fornecer maior eficiência econômica na produção da celulose kraft.

O objetivo principal do presente trabalho foi a análise de custo e efeito na qualidade da polpa na diminuição da água fresca no processo de branqueamento com a utilização da prensa como o equipamento que viabiliza a maior eficiência na lavagem, facilitando o fechamento de circuito de água.

\section{EXPERIMENTAL}

Foi utilizada uma polpa celulósica industrial de Eucalyptus spp. pré-deslignificada com oxigênio, com alvura de $62,5 \%$ ISO. O branqueamento foi realizado através da sequência $\mathrm{D}_{0}(\mathrm{E}+\mathrm{P}) \mathrm{D}_{1} \mathrm{P}$. Utilizou-se quatro amostras idênticas e para cada uma foi proposta 10 lavagens diferentes viabilizando a redução de água, totalizando 40 amostras finais. $\mathrm{O}$ branqueamento foi realizando utilizando sempre as mesmas condições em cada estágio com o objetivo de atingir a alvura de $92 \pm 0,5 \%$ ISO (Tabela 1) e de simular o fechamento de circuito realizado na indústria. O branqueamento foi efetuado em sacos de polietileno, com amostras de $150 \mathrm{~g}$ a.s. (absolutamente seca) de polpa. $\mathrm{O}$ licor de branqueamento foi adicionado à polpa nas condições apresentadas na Tabela 1 . Após a mistura em saco de polietileno, a polpa foi aquecida em microondas e transferida para um banho de vapor termostatizado, onde foi mantida pelo tempo pré-estabelecido.

Tabela 1 - Condições gerais de branqueamento

\begin{tabular}{|c|c|c|c|c|}
\hline \multirow{2}{*}{ Condições } & \multicolumn{4}{|c|}{ Estágio de Branqueamento } \\
\hline Consistência, $\%$ & 10 & 10 & 10 & 10 \\
\hline Temperatura, ${ }^{\circ} \mathrm{C}$ & 60 & 70 & 70 & 90 \\
\hline Tempo $(\mathrm{min})$ & 30 & 60 & 180 & 120 \\
\hline $\mathrm{H}_{2} \mathrm{O}_{2}(\mathrm{~kg} / \mathrm{t})$ & - & 3,0 & - & 3,0 \\
\hline $\mathrm{NaOH}(\mathrm{kg} / \mathrm{t})$ & - & 10,0 & - & 10,0 \\
\hline $\mathrm{H}_{2} \mathrm{SO}_{4}(\mathrm{~kg} / \mathrm{t})$ & 2,0 & - & 2,0 & - \\
\hline $\mathrm{ClO}_{2}(\mathrm{~kg} / \mathrm{t})$ & 18,8 & - & 10,0 & - \\
\hline
\end{tabular}

Terminada a reação, foram extraídas amostras de licor residual para análises de pH, DQO e cor aparente. As polpas das quatro amostras foram lavadas com um fator de 9, 6, 3 e $0 \mathrm{~m} 3 / \mathrm{t}$ de polpa, respectivamente, e logo depois centrifugadas para a coleta dos efluentes de cada etapa do branqueamento de maneira semelhante a prensa industrial (Figura 1). 


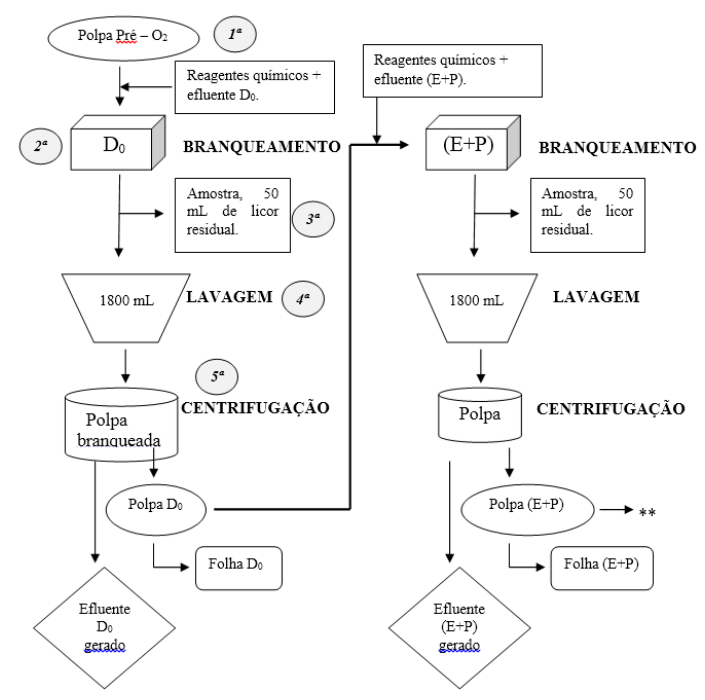

Figura 1 - Etapas realizadas em cada estágio de branqueamento.

A consistência da polpa foi ajustada para 10\%, nos quatros estágios de branqueamento, através da adição do efluente gerado nas sequências feitas anteriormente em cada processo de branqueamento. Junto com o efluente foram adicionados os reagentes químicos de cada estágio como apresentado na Tabela 1. O branqueamento com quatro amostras e suas respectivas lavagens propondo à redução de água, foi realizado dez vezes utilizando sempre as mesmas condições do processo. A realização das repetições do branqueamento utilizando o efluente da sequência anterior foi uma simulação do fechamento de circuito realizado na indústria.

No final de cada um dos estágios foi realizada a lavagem da polpa celulósica com quantidades diferentes $\left(9,6,3\right.$ e $\left.0 \mathrm{~m}^{3} / \mathrm{t}\right)$ de efluente/efluente ou efluente/água fresca nas quatro amostras. A lavagem visou ter reaproveitar o efluente gerado em cada sequência anterior de branqueamento. Dentre as quatro amostras com diferentes lavagens, os dois primeiros estágios de branqueamento - $\mathrm{D}_{0}$ e $(E+P)$ - das sequências da amostra com lavagem de $9 \mathrm{~m}^{3} / \mathrm{t}(1800 \mathrm{~mL})$ foi demonstrada na Figura 1. A polpa gerada no estágio do branqueamento $\mathrm{D}_{0}$ (como exemplo) continua no estágio posterior $(\mathrm{E}+\mathrm{P})$ até o final da sequência de branqueamento (D1 e P) com a quantidade de $1800 \mathrm{~mL}$ de água de lavagem. Conforme a Figura 1, as outras três amostras de polpa foram feitas da mesma maneira, mas com a redução da quantidade de água de lavagem de 6 m³/t $(1200 \mathrm{~mL}), 3 \mathrm{~m} / \mathrm{t}(600 \mathrm{~mL})$ e $0 \mathrm{~m}^{3} / \mathrm{t}$ e foram distribuídas de acordo com a Tabela 2.

Na primeira sequência de branqueamento, foram realizados os estágios conforme a Tabela 1, com água destilada tanto para o ajuste de consistência quanto para a lavagem da polpa. Após a geração dos efluentes nesta primeira sequência de branqueamento, começou a ser feita a reutilização dos efluentes gerados em cada estágio de branqueamento para as próximas sequências.

$\mathrm{Na}$ lavagem do estágio $\mathrm{D}_{0}$ com $9 \mathrm{~m}^{3} / \mathrm{t}(1800 \mathrm{~mL})$ a água de lavagem foi dividida em $900 \mathrm{~mL}$ de efluente $\mathrm{D}_{0}$ e $900 \mathrm{~mL}$ de efluente $\mathrm{D}_{1}$ dos respectivos estágios da sequência anterior de branqueamento e assim, consecutivamente até o estágio $\mathrm{P}$ (Tabela 2). Este procedimento foi repetido para as lavagens com 6 e $3 \mathrm{~m}^{3} / \mathrm{t}$. 
Tabela 2 - Distribuição do volume de águas de lavagem em cada estágio do branqueamento

\begin{tabular}{|c|c|c|c|c|c|c|c|}
\hline \multirow{2}{*}{ ESTÁGIOS } & \multicolumn{7}{|c|}{ LAVAGEM } \\
\cline { 2 - 9 } & $900 \mathrm{~mL} / \mathrm{t}(1800 \mathrm{~mL})$ & \multicolumn{2}{|c|}{$6 \mathrm{~m}^{3} / \mathrm{t}(1200 \mathrm{~mL})$} & $3 \mathrm{~m}^{3} / \mathrm{t}(600 \mathrm{~mL})$ & $0 \mathrm{~m}^{3} / \mathrm{t}$ \\
\hline $\mathrm{D}_{0}$ & $\mathrm{D}_{0}$ & $\mathrm{D}_{1}$ & $\mathrm{D}_{0}$ & $\mathrm{D}_{1}$ & $\mathrm{D}_{0}$ & $\mathrm{D}_{1}$ & - \\
\hline$(\mathrm{E}+\mathrm{P})$ & $(\mathrm{E}+\mathrm{P})$ & $\mathrm{P}$ & $(\mathrm{E}+\mathrm{P})$ & $\mathrm{P}$ & $(\mathrm{E}+\mathrm{P})$ & $\mathrm{P}$ & - \\
\hline $\mathrm{D} 1$ & $\mathrm{D} 1$ & Água & $\mathrm{D} 1$ & Água & $\mathrm{D} 1$ & Água & - \\
\hline $\mathrm{P}$ & $\mathrm{P}$ & Água & $\mathrm{P}$ & Água & $\mathrm{P}$ & Água & - \\
\hline
\end{tabular}

\subsection{Cálculos do Payback}

A ferramenta utilizada para o cálculo referido é o Microsoft Excel utilizando a função de cálculo financeiro NPER, onde retorna o número de períodos de um investimento com base em pagamentos constantes periódicos e uma taxa de juros constante. Os custos gerados pela utilização contínua de água fresca, sem a utilização do fechamento de circuito é observada na Tabela 4 e foram provenientes de uma indústria de celulose branqueada.

Tabela 3 - Custo da água no processo de branqueamento

\begin{tabular}{|c|c|}
\cline { 2 - 2 } \multicolumn{1}{c|}{} & \multicolumn{1}{c|}{ Custos $(\mathrm{R} \$)$} \\
\hline Captação e tratamento da água & $1,21 / \mathrm{tsa}^{*}-62,00 / \mathrm{mil} \mathrm{m}^{3}$ \\
\hline Desmineralização da água & $0,56 / \mathrm{tsa}-0,45 / \mathrm{m}^{3}$ \\
\hline Tratamento do efluente & $7,17 / \mathrm{tsa}-330,00 / \mathrm{mil} \mathrm{m}^{3}$ \\
\hline tsa $=$ tonelada de polpa absolutamente seca. &
\end{tabular}

O fechamento de circuito é o melhor método para diminuir a quantidade de água utilizada pelas indústrias de branqueamento de celulose. $\mathrm{O}$ cálculo do payback foi realizado para a sequência com $9 \mathrm{~m}^{3} / \mathrm{t}$ de água fresca, utilizado pela maioria das indústrias, e $\operatorname{com~} 3 \mathrm{~m}^{3} / \mathrm{t}$ referentes ao presente estudo.

\subsection{Estudo da redução de água fresca no branqueamento}

Para a comparação e a apresentação do custo relacionado à água do processo de branqueamento apresenta-se os seguintes casos: Caso 1 - processo usual onde se utilizam os equipamentos filtro lavador e difusor. Caso 2 - processo proposto onde se utilizam prensas.

Utilizando a função de cálculo financeiro NPER (número de períodos para alcançar o montante investido), onde retorna o número de períodos (1) de um investimento (2) com base em pagamentos constantes periódicos (3) e uma taxa de juros constante (4), ou seja: Investimento (2): aproximadamente $\mathrm{R} \$ 30.000 .000,00$ (prensa) e Redução anual de custos com água (3): $\mathrm{R} \$$ 3.687.960,00 (conforme Tabela 3).

Considerando a taxa de juros (4) (TMA adotado) de 5,08 \% a.a. pode-se calcular o número de períodos (1) em anos como vemos a seguir: 
Onde NPER (1) é o número de períodos para alcançar o montante investido; Taxa (4) é a taxa de juros por período; Pgto (3) é o pagamento efetuado a cada período; Vp (2) é o valor presente ou a quantia total do montante investido. Sendo assim, com a utilização da Equação 1, obteremos o resultado da Equação 2:

\section{NPER $=(5,08 \% ; 3687960 ; 30000000)$}

(Equação 2)

\section{RESULTADOS E DISCUSSÃO}

Na Figura 2 têm-se a análise da DQO das dez sequências com a lavagem de $9 \mathrm{~m}^{3} / \mathrm{t}$. Os resultados de DQO foram obtidos com uma diferença muito grande entre a primeira e última sequência de branqueamento com a mesma lavagem e com um princípio de estabilização da DQO para as três últimas sequências, resultados semelhantes foram encontrado por Costa et al. (2006). Em decorrência da diminuição de água de lavagem da polpa de celulose no experimento, a DQO tendeu a aumentar como mostrada na Figura 2.

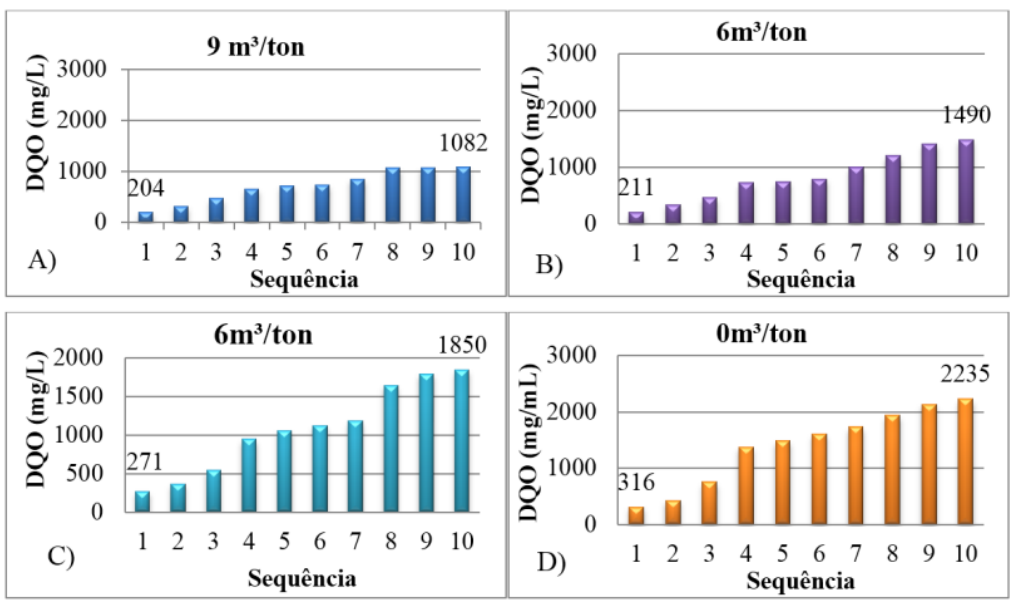

Figura 2 - Resultado da DQO nas dez sequências com lavagem de $9 \mathrm{~m}^{3} / \mathrm{t}(\mathrm{A}), 6 \mathrm{~m}^{3} / \mathrm{t}(\mathrm{B}), 3 \mathrm{~m}^{3} / \mathrm{t}(\mathrm{C})$, e $0 \mathrm{~m}^{3} / \mathrm{t}(\mathrm{D})$ de polpa.

A diferença entre a Figura 2A e a Figura 2D, mostra que lavando a polpa com $6 \mathrm{~m}^{3} / \mathrm{t}$ há o aumento do carry over quando comparado a lavagem utilizando $9 \mathrm{~m}^{3} / \mathrm{t}$, pois o efluente do branqueamento é rico em matéria orgânica e quanto menor for à quantidade de água de lavagem, maior será seu acúmulo. Valores da DQO de efluentes de branqueamento antes da Estação de Tratamento de Efluentes de modernas fábricas de celulose kraft de eucalipto, segundo Mounteer (2005), variam de 500 a $1500 \mathrm{mg} / \mathrm{L}$, dependendo do tipo de branqueamento ("standard", Livre de Cloro Molecular- ECF ou Totalmente Livre de Cloro Molecular- $T C F$ ) e da dosagem dos reagentes utilizados. Esta DQO está de acordo com os resultados encontrados, onde a variação destes resultados com as polpas lavadas com água fresca (9 e $6 \mathrm{~m}^{3} / \mathrm{t}$ de polpa), variaram de 211 a $1490 \mathrm{mg} / \mathrm{L}$, estando dentro de valores praticados nas indústrias de celulose. 
Os resultados da DQO do efluente com as sequências de branqueamento sem lavagem entre os estágios (Figura 2D), mostra que após a quinta sequência de branqueamento a DQO chegou a 1500 $\mathrm{mg} / \mathrm{L}$, gerando um grande acúmulo de matéria orgânica nas sequências dificultando o fechamento de circuito. Este valor de $1500 \mathrm{mg} / \mathrm{L}$ são valores de DQO praticados por indústrias de celulose segundo Mounteer (2005).

Na Figura 3, observa-se a alvura final das polpas, no último estágio de branqueamento. Na Figura 3A, observa-se que a primeira até a última sequência de branqueamento houve um decréscimo de $\Delta 3,7 \%$ ISO na alvura. Isso ocorreu pelo fato de ser o último estágio do branqueamento, onde a polpa está próxima ao 90\% ISO e esta alta alvura é mais sensível a matéria orgânica.

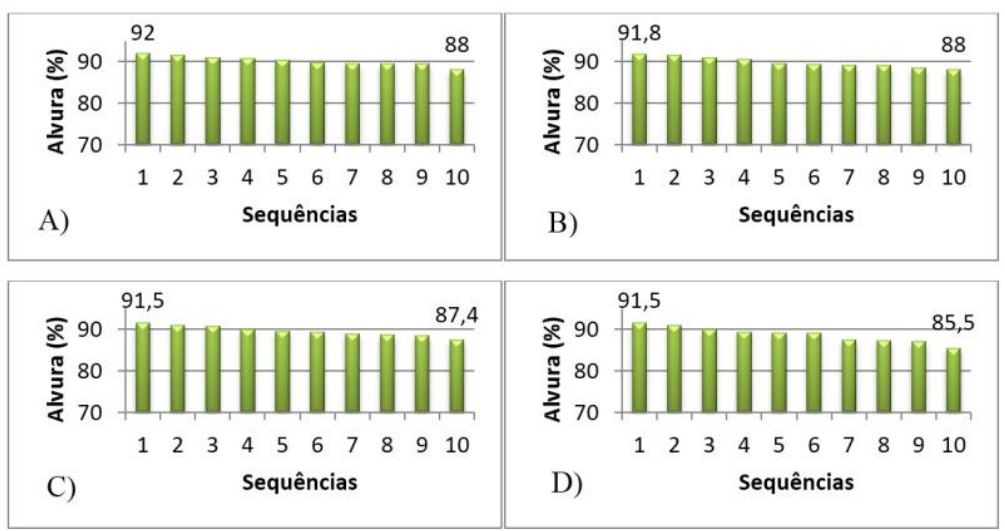

Figura 3 - Alvura final para amostras com lavagens de $9 \mathrm{~m}^{3} / \mathrm{t}(\mathrm{A}), 6 \mathrm{~m}^{3} / \mathrm{t}(\mathrm{B}), 3 \mathrm{~m}^{3} / \mathrm{t}(\mathrm{C}), \mathrm{e} 0 \mathrm{~m}^{3} / \mathrm{t}$ (D) de polpa.

A queda mais brusca da alvura aconteceu na Figura 3D onde não ocorreu a lavagem, mostrando que a quantidade de matéria orgânica acumulada nas dez sequências, fez com que a alvura alcançada fosse inferiores as demais. A alvura do último estágio de branqueamento quando a lavagem foi realizada com $3 \mathrm{~m}^{3} / \mathrm{t}$, mostrado na Figura $3 \mathrm{C}$, de aproximadamente 87,5\%ISO, ainda é aceitável por muitos clientes. Já a polpa que não obteve nenhuma lavagem durante as dez sequência de branqueamento não poderia ser comercializada, pois não está dentro dos parâmetros exigidos pelo mercado.

Com o aumento da DQO do efluente, mostrado na Figura 4, nota-se o aumento da reversão de alvura quando a mesma sequência foi feita novamente utilizando o efluente da sequência anterior. Com a lavagem de $9 \mathrm{~m}^{3} / \mathrm{t}$ e a utilização de prensas, há menor quantidade de matéria orgânica na polpa final de cada sequência do ciclo, o que causa menor reversão da alvura, quando comparado aos outros estágios de branqueamentos. 


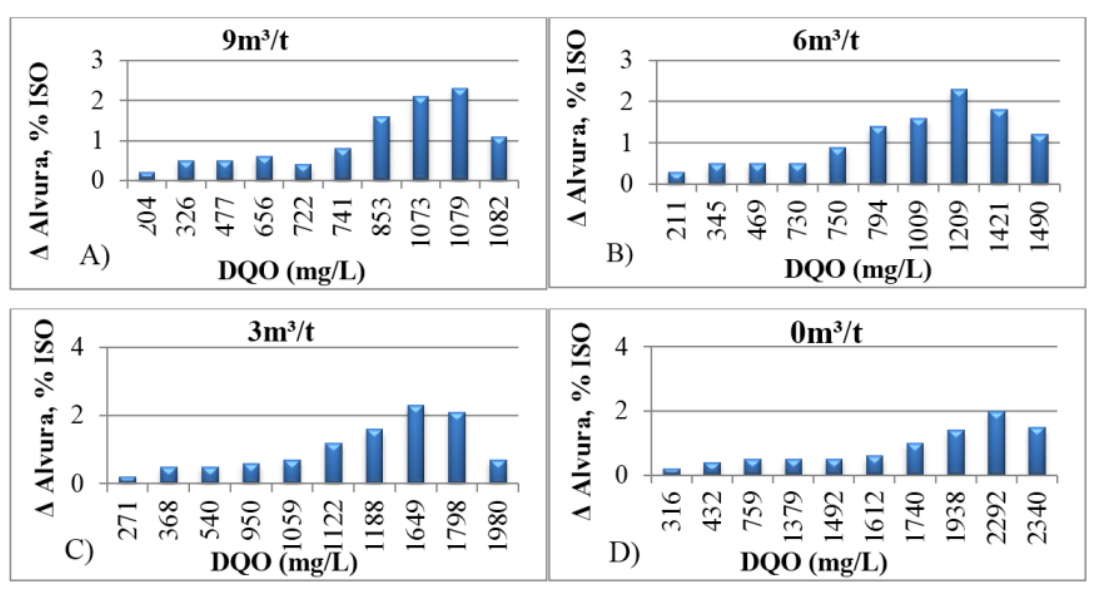

Figura 4 - Variação da alvura após a reversão de alvura na polpa final comparada a DQO.

Um fator ligado a reversão de alvura é quando são utilizados reagentes incapazes de remover toda a lignina da polpa ou a deficiência na retirada desta lignina, que pode ser resultados de lavagem insuficiente (Suess e Leporini Filho, 2005). Apesar do aumento da reversão observado, os dados estão dentro dos parâmetros encontrados por Milagres et al. (2011) que cita, sobre uma sequência similar ao da presente pesquisa - $\mathrm{D}(\mathrm{EPO}) \mathrm{DP}_{2}$ - que obteve uma variação de $\Delta 1,8$ a $\Delta 2,0 \%$ ISO.

No presente trabalho, a variação das diferentes lavagens de $9,6,3$ e $0 \mathrm{~m}^{3} / \mathrm{t}$, ocorreu entre $\Delta 0,2$ a $\Delta 2,3 \%$ ISO, com a média de $\Delta 1,25 \%$ ISO que se aproxima dos dados da pesquisa de Eiras et al. (2005) feita com a sequência OD(PO)DP, que teve a reversão de alvura média de $\Delta 1,5 \%$ ISO também citada por Oliveira et al. (2006) e por Pimenta (2010) que fez a comparação laboratorial ( $\Delta 1,5 \%$ ISO) com o fabril ( $\Delta 2,1 \%$ ISO).

A Figura 4D que indica as sequências que não obtiveram lavagens estão dentro dos parâmetros considerados na literatura, pois obtiveram média de $\Delta 1,1 \%$ ISO. Para Ferreira et al. (2006) a reversão de alvura variou $\Delta 1,3$ a $\Delta 2,5 \%$ ISO, sendo a média de $\Delta 2,0 \%$, valor este que pode ser considerado muito satisfatório para polpa kraft de eucalipto.

Vários estudos foram realizados para a análise das principais causas da reversão de alvura. Conforme Eiras et al. (2005) e Oliveira et al. (2006), possuem diversos indicativos que causam a reversão de alvura, como por exemplo, os grupos carbonilas, oxidação dos carboidratos, extrativos, ácido hexanurônico e metais $(\mathrm{Fe})$. Neste trabalho podemos verificar que a DQO também tem efeito significativo na reversão de alvura já que afetam a branqueabilidade e a estabilidade de alvura da polpa (Costa et al., 2003).

\subsection{Resultados do Payback}

Com a possível redução de água fresca no processo de branqueamento, diminuição dos gastos com a água e a implantação de uma prensa no valor de $\mathrm{R} \$ 30.000 .000,00$, os resultados foram analisados para a produção de 2.000 t/dia para ambos os casos. Aplica-se a quantidade de 
$9 \mathrm{~m}^{3} / \mathrm{t}$ no caso 1 e a redução para $3 \mathrm{~m}^{3} / \mathrm{t}$ no caso 2 . A comparação do custo da água pura e tratada relacionada aos dois casos estão indicadas na Tabela 4.

Tabela 4 - Comparação dos dois casos

\begin{tabular}{|l|l|l|l|l|}
\hline & \multicolumn{1}{|c|}{$\begin{array}{c}\text { Captação e } \\
\text { tratamento da água }\end{array}$} & $\begin{array}{c}\text { Desminerali- } \\
\text { zação da água }\end{array}$ & $\begin{array}{l}\text { Tratamento } \\
\text { do efluente }\end{array}$ & $\begin{array}{c}\text { Total Parcial } \\
\text { (R\$) }\end{array}$ \\
\hline CUSTO (R\$) & $0,062 / \mathrm{m}^{3}$ & $0,45 / \mathrm{m}^{3}$ & $0,33 / \mathrm{m}^{3}$ & \\
\hline CASO 1 $\left(18 \mathrm{mil} \mathrm{m}^{3} / \mathrm{dia}\right)$ & $\mathrm{R} \$ 1.116,00$ & $\mathrm{R} \$ 8.100,00$ & $\mathrm{R} \$ 5.940,00$ & $15.156,00 /$ dia \\
\hline CASO 2 $\left(6 \mathrm{mil} \mathrm{m}^{3} / \mathrm{dia}\right)$ & $\mathrm{R} \$ 372,00$ & $\mathrm{R} \$ 2.700,00$ & $\mathrm{R} \$ 1980,00$ & $5.052,00 /$ dia \\
\hline Diferença entre Caso 1 e Caso 2 & & $10.104,00 /$ dia \\
\hline \multicolumn{2}{|l|}{ Diferença entre Caso 1 e Caso 2 (anual considerando 365 dias) } & $3.687 .960,00$ \\
\hline
\end{tabular}

Com a utilização da Equação 1 e com os dados da Tabela 4, o resultado encontrado com o payback foi que o número de períodos para alcançar o montante investido é de 6,98 , ou seja, o retorno do investimento considerando apenas a economia da redução do uso de água fresca no processo é de aproximadamente 7 anos.

\section{CONCLUSÕES}

É possível obter uma real redução do consumo de água fresca no processo de branqueamento da polpa celulósica sem uma grande perda de qualidade da polpa branqueada. A alvura de cada estágio de branqueamento, dentre uma mesma lavagem e nas diferentes lavagens, diminuíram gradativamente. A reversão de alvura sofreu um grande efeito do aumento da DQO no efluente do branqueamento. Neste trabalho foi constatado que realizar o branqueamento sem lavagem entre os estágios de branqueamento, mesmo com uso da prensa, é inviável até o momento. A redução de água de $9 \mathrm{~m}^{3} / \mathrm{t}$ até $3 \mathrm{~m}^{3} / \mathrm{t}$ foi constatado que é viável pela qualidade final da polpa e também pelo tempo de retorno do investimento realizado na planta de branqueamento com a implantação da prensa.

\section{AGRADECIMENTOS}

Os autores agradecem ao $\mathrm{CNPq}$, pela bolsa concedida.

\section{REFERÊNCIAS}

ALMEIDA, A. M.; PINHEIRO, A. R. O.; CARNEIRO, R., SILVA, R. C. Processos Limpos no Branqueamento de Polpa Celulósica. 2008. Faculdade de Telêmaco Borba (FATEB), Engenharia Química - Ênfase em Celulose e Papel, Telêmaco Borba PR, 2008.

COSTA, M. M.; COLODETTE, J. L.; LANDIM, A.; SILVA, C. M.; CARVALHO, A. M. M. L. Nova tecnologia de branqueamento de celulose adaptada ao fechamento do circuito de água. Rev. Árvore, v.30, n.1, p.129-139, 2006. 


\section{9 a 22 de outubro de 2014 \\ Florianópolis/SC}

EIRAS, K. M. M., COLODETTE, J. L., CARVALHO, A. M. M. L. Estudo das causas de reversão de alvura de polpas Kraft de Eucalipto. Cerne, v. 11, n. 4. p. 354-368, 2005.

FERREIRA, C. R.; FANTINI JUNIOR, M.; COLODETTE, J. L.; OLIVEIRA, R. C.; GOMIDE, J. L.; CARVALHO, A. M. M. L. Avaliação tecnológica de clones de eucalipto: parte 2 - qualidade da celulose branqueada Kraft para papel de imprimir e escrever. Sci. Forest. n. 71, p. 9-18, 2006.

KASSAI, J.R.; KASSAI, R.; SANTOS, A. Retorno de Investimento: Abordagem Matemática e Contábil do Lucro Empresarial, São Paulo: Editora Atlas, 2000.

MILAGRES, F. R. COLODETTE J.L.; RABELO M.S.; CARVALHO D. M.; Branqueamento de polpa de eucalipto com sequências curtas. Ciên. Florest., v. 21, n. 1, p. 159$166,2011$.

MOUNTEER, A. H. Tratabilidade comparativa de filtrados de branqueamento ECF e TCF de polpa kraft de eucalipto. $11 \mathrm{f}$. Departamento de Engenharia Civil, Universidade Federal de Viçosa, 2005. Disponível em: http://www.eucalyptus.com.br/icep01/ann_mounteer.pdf. Acesso em: 10 abr 2012.

OLIVEIRA, R. L.; COLODETTE, J. L.; EIRAS, K. M. M.; VENTORIM, G. The effect of Wood supply and bleaching process on pulp brightness stability. Rev. Árvore, v.30, n.3, p.439450, 2006.

PIMENTA, L. M. Aperfeiçoamento da sequência O/OD*(EP)DP para branqueamento de celulose Kraft de Eucalipto. 2010. 30 f. Dissertação (Mestrado), Universidade Federal de Viçosa. Viçosa - MG, 2010.

RANGANATHAN, K.; JEYAPAUL, S.; SHARMA, D. C. Assessment of water pollution in different bleaching based paper manufacturing and textile dyeing industries in India. Env. Monit. Asses. v. 134, n. 1, p. 363-372, 2007.

SUESS, H. U.; LEPORINI FILHO, C. Progress in bleaching to top brightness with low reversion. Papel, v. 66, n. 2, p.59-67, 2005.

THAKORE A, OEI J, HEIMBURGER S. Significant improvements in ECF bleaching using a minimal capital approach to better filtrate management: Lowering bleaching costs through modified filtrate recycling. Pulp Pap. Can. v.102, n.9, p.40-43, 2001. 